\title{
Introduction to Melatonin: An Endogenously Synthesized Super-Compound
}

\author{
Nilutpal Sharma Bora* \\ Department of Pharmaceutical Sciences, Dibrugarh University, Dibrugarh, Assam, India \\ *Corresponding Author: Nilutpal Sharma Bora, Department of Pharmaceutical Sciences, Dibrugarh University, Dibrugarh, Assam, India.
}

Received: April 17, 2019; Published: May 10, 2019

DOI: $10.31080 /$ ASPS.2019.03.0280

As the title suggests, melatonin (synonym: N-acetyl-5methoxytryptamine) is an endogenous substances which is synthesized by the pineal gland in the human brain and is known to regulate various physiological and pathological processes. Melatonin is known to regulate the circardian rhythms and sleep processes in diurnal species, with concentrations remaining low during the day and reaching optimal levels at night. Melatonin after synthesis is rapidly absorbed into the blood stream and thereafter into various bodily fluids. Seasonal and age related variations in the content of melatonin in the human body is observed; with increased concentration in winters than in summers and decreased synthesizing capacity with an increase in age. Various clinical and pre-clinical toxicity analysis of melatonin has proved that it has no evidence of acute or chronic toxicity [1].

Melatonin is known to a potent antioxidant and has been found to increase the activity of endogenous antioxidant enzymes like glutathione peroxidase and superoxide dismutase. It has been found to be more potent than glutathione and mannitol in hydroxyl radical scavenging activity. Melatonin has also been found to decrease the activity of nitric oxide synthase (NOS) which is a pro oxidative enzyme. This antioxidant potential of melatonin has facilitated its use as a radioprotectant against both ionizing and non-ionising radiation; as radiation-induced cellular injury is attributed mainly to ROS. Melatonin has been found to be an effective supplement in radiation therapy as it ameliorates oxidative stress caused by gamma radiation by stimulating glutathione, modulating gamma-glutamyltransferase activity and by exerting a hypolipidemic effect. Melatonin has also shown promising ultraviolet (UV) protective effect when used in combination with vitamins $\mathrm{E}$ and $\mathrm{C}$ in a human study, in vivo. Topically applied melatonin has been found to be the most potent antioxidative defense systems against skin aging induced by UV radiation; as it the most common environmental factor which contributes to skin aging. Clinically, exogenous melatonin is more effective when used via topical route rather than oral, since orally administered melatonin appears in rather low levels in the blood due to prominent first-pass degradation in the liver [2-4].

\section{Bibliography}

1. Shirazi A., et al. "A Radiobiological Review on Melatonin: A Novel Radioprotector". Journal of Radiation Research 48 (2007): 263-272.

2. Dreher Gabard., et al. "Topical melatonin in combination with vitamins $\mathrm{E}$ and $\mathrm{C}$ protects skin from ultraviolet-induced erythema: a human study in vivo". British Journal of Dermatology 139 (1998): 332-339.

3. El-Missiry MA., et al. "Ameliorative effect of melatonin against gamma-irradiation-induced oxidative stress and tissue injury". Ecotoxicology and Environmental Safety 66 (2007): 278-286.

4. Kleszczynski K and Fischer TW. "Melatonin and human skin aging". Dermatoendocrinology 4 (2012): 245-252.

Volume 3 Issue 6 June 2019

(C) All rights are reserved by Nilutpal Sharma Bora. 